\title{
STARS AND
}

\section{CIRCUMSTELLAR ENVELOPES}




\title{
COOL STAR MODELS
}

\author{
UFFE GRÅE JØRGENSEN \\ Niels Bohr Institute, Copenhagen University Observatory \\ Juliane Maries Vej 30, DK-2100 Copenhagen, Denmark
}

\begin{abstract}
It is the intention with the present review to summarize the role of molecules in cool stars. Which molecules do we find there, which are the most important ones, what do we know about them, what more would we like to know ? - and what are the current main problems and progresses we meet when including molecules in models of cool stars?
\end{abstract}

\section{Introduction}

Molecules are found in a wide range of stars, from our own Sun to much cooler objects as red giants and Brown Dwarfs. The light from our own Sun is so bright that spectral lines from molecules which constitute only trace elements can be identified. Indeed half of all the molecules known in stars are seen in the solar spectrum, although our Sun is not a typical "molecule dominated" object. The spectrum and the photospheric structure of red giant stars, on the other hand, are completely dominated by molecular processes. These stars are huge complex dynamical systems, producing about half of all the elements which surround us in nature. The cool mainsequence dwarf stars represent another important type of objects where molecules are the dominant constituent. They are the most numerous stars in the universe; about $3 / 4$ of all stars are cool main-sequence stars. Brown Dwarfs are also dominated by molecules. They are gas systems that became neither planets nor stars. Finally we find the cool white dwarfs, which are electron-degenerate objects that once - in the very beginning of the Universe - were nuclear burning stars, but now are compact remnants slowly radiating their left-over energy into the Universe. Their molecules may help us reveal the age of the Universe. All these diverse classes of objects have only little more in common than their low temperatures. 
In the gas envelopes surrounding the giant stars the temperature may drop to interstellar values, and here large complex molecules may form, which is the subject of the contribution by Olofsson to this volume. In the present paper I will describe the photospheric layers, which is where most of the near ultraviolet, the visual, and the near infrared spectra originate. Many molecules build up in these layers, but most of them are radicals of only two atoms, which is due to the relatively high temperatures (typically $1000-4000 \mathrm{~K}$ ) and the low gas pressures (down to, say, $10^{-10}$ atmosphere).

Existing grids and general problems and progress in the modelling of late-type photospheres were reviewed recently by Johnson $(1986,1991)$ and by Gustafsson \& Jørgensen (1994). Determination of fundamental stellar parameters from stellar spectra was summarized by Gustafsson \& Jørgensen (1985), and chemical analysis of cool stars was reviewed by Gustafsson (1989). Existing atomic data bases for stellar atmosphere computations were reviewed by Martin (1992) and by Kurucz (1994), and molecular data bases were described by Jørgensen (1995). Systematic descriptions of individual molecules in stars can be found in Spinrad \& Wing (1969), Jaschek \& Jaschek (1987, 1995) and Tsuji (1986). Recent conferences that dealt with aspects of molecules in stars include IAU Symp. 150, Astrochemistry of Cosmic Phenomena, Brazil 1991, IAU Coll. 146, Molecules in the Stellar Environment, Denmark 1993 (Jørgensen 1994a), Laboratory and Astronomical High Resolution Spectra, Belgium 1994 (Sauval et al. 1995), The Bottom of the Main Sequence and Beyond, Germany 1994 (Tinney 1995), IAU Symp. 177, The Carbon Star Phenomenon, Turkey 1996 (Wing 1996).

\section{Molecules in stars}

While continuum processes and atomic spectral lines $(b-b$ transitions) are responsible for the interaction between radiation and matter in the atmosphere of hot stars, molecules are of increasing importance for successively cooler stars. In the solar spectrum, about $20 \%$ of the spectral lines in the visible region are due to molecules. The solar photosphere itself has an effective temperature of $5780 \mathrm{~K}$ and resembles the spectrum of a G2V star, whereas the sunspots are about $2000 \mathrm{~K}$ cooler and their spectra resemble those of K5V stars. Indeed the Sun is in this sense a bright nearby star of two different classes, and about half of the $\approx 50$ molecules which have been identified in the spectrum of (the photosphere of) any star, have been identified in the sunspot spectra too. In Table 1 all molecules are listed which have been identified in stars (including the Sun). It is seen that many more molecules are identified in the Sun than in G and K type stars. This, of course, suggests that with higher resolution, better sensitivity, and larger telescopes, many more molecules could be identified in stars of type $\mathrm{G}$ and 
$\mathrm{K}$ - as they were identified in the photosphere and spots of the Sun due to the better $\mathrm{S} / \mathrm{N}$ ratio and resolution in the solar spectra.

For stars of effective temperatures $\lesssim$ the sunspot temperature (i.e., $\mathrm{T}_{\text {eff }}$ $\lesssim 3500 \mathrm{~K}$ ), molecules completely dominate the opacity. Stellar atmospheres of such effective temperatures can be modelled (with high accuracy) without taking the atomic lines into account at all. For most models, $\mathrm{H}^{-}$plays an important role for the continuum formation. Since the minimum absorption of $\mathrm{H}^{-}$is at $1.6 \mu \mathrm{m}$ and the Planck maximum is between $0.8 \mu \mathrm{m}$ (for $\mathrm{T}=3500 \mathrm{~K}$ ) and $1.6 \mu \mathrm{m}$ (for $\mathrm{T}=1800 \mathrm{~K}$ ), all cool stars have a strong maximum flux "plateau" in the 1-2 $\mu \mathrm{m}$ region. Absorption close to this region is therefore of particularly great importance for the energy balance. At deeper layers in the stellar atmosphere the gas temperature is higher than the effective temperature, and the maximum flux will be at shorter wavelengths, while the temperature near the surface layers may be as low as $\approx 1000 \mathrm{~K}$, giving rise to more long-wavelength radiation. Absorption in the near infrared might therefore dominate the upper atmospheric structure, while more blue absorption systems will influence the structure of the deeper layers.

Chemically, stars are divided into those with $\mathrm{C} / \mathrm{O}>1$ (carbon stars), those with $\mathrm{C} / \mathrm{O} \approx 1$ (S-type stars), and those with $\mathrm{C} / \mathrm{O}<1$ (mainly Mtype stars when we talk about molecules, but also the somewhat warmer counterparts of spectral type $\mathrm{K}$ and $\mathrm{G}$ ). We therefore list molecules in these three types of objects (as well as the Sun because of the far better spectra available of the Sun) separately in Table 1 . The pronounced difference between spectra of these three chemical classes of objects is caused by the high dissociation energy of the $\mathrm{CO}$ molecule, which ensures that all carbon is bound into $\mathrm{CO}$ if $\mathrm{C} / \mathrm{O}<1$ and all oxygen is bound in $\mathrm{CO}$ if $\mathrm{C} / \mathrm{O}>$ 1. The leftover oxygen (for G, K, M stars) and the leftover carbon (for $\mathrm{C}$ stars), respectively, cause a completely different spectral appearance of the $\mathrm{C}$ and $\mathrm{M}$-type stars. $\mathrm{TiO}, \mathrm{SiO}$ and $\mathrm{H}_{2} \mathrm{O}$ dominate the M-type spectrum, whereas $\mathrm{C}_{2}, \mathrm{CN}, \mathrm{CH}, \mathrm{HCN}$, and $\mathrm{C}_{2} \mathrm{H}_{2}$ dominate the C-type spectrum. In the spectrum of S-type stars there are neither free carbon nor oxygen atoms left after the $\mathrm{CO}$ formation, and most of the nitrogen is bound in the nonpolar $\mathrm{N}_{2}$ molecule. This means that the three most abundant atoms (after hydrogen and helium) are virtually absent from the opacity in S stars, and much less abundant molecules will therefore dominate the spectrum, including $\mathrm{LaO}, \mathrm{YO}, \mathrm{ZrO}$, and various sulfides and hydrides. No polyatomic molecules are known to be present in the spectrum of S-type stars, which presumably will cause the atmosphere of S-type stars to be far more compact (i.e., geometrically smaller and with higher gas pressure gradient) than the atmosphere of corresponding $\mathrm{M}$ and C-type stars. This is because the polyatomics (in the $\mathrm{M}$ and C-type stars) form at lower temperatures than 
the diatomics, and therefore at shallower depths where the atmosphere is more easily expandable by the radiative force.

Through its influence on the density of the gas, gravity, too, plays an important role in the formation of molecules. The investigations of dwarf and giant model atmospheres have taken two very different directions during recent years, which will be described separately below. Here only a few general comments about molecules in dwarfs and giants will be given. Diatomic hydrides are usually more abundant in dwarfs than in giants, and their abundances relative to neutral atomic lines have been used quantitatively as a measure for the stellar gravity (e.g., Bonnell \& Bell 1993). Pairs of molecules which respond oppositely to gravity can have such strong effects on the spectrum that they influence even the stellar colours. An example of this effect is the bifurcation between cool M-type dwarfs and giants in the $\mathrm{H}-\mathrm{J}$ versus $\mathrm{H}-\mathrm{K}$ diagram (Bessell \& Brett 1988) which was recently explained (Jørgensen \& Wing 1996) as a negative gravity sensitivity of the $\mathrm{CO}$ spectral bands (i.e., decreasing band intensity as function of increasing gravity) combined with a positive gravity sensitivity of the $\mathrm{H}_{2} \mathrm{O}$ bands and the $\mathrm{H}^{-}$opacity. We list in Table 1 the gravity-sensitivity of some of the molecules by noticing whether the bands are stronger in giants than in dwarfs $(\mathrm{g}+)$ or visa versa $(\mathrm{d}+)$. The note $d w$ means that the band has been observed in dwarfs only, which indicates an even stronger (positive) gravity sensitivity.

The sensitivities of the bands to temperature are not listed in Table 1. Usually the molecules have a well defined maximum band intensity at a given spectral type (i.e., given effective temperature for given luminosity), which is described in detail by Jaschek \& Jaschek (1987). Generally stronger polyatomics and weaker diatomic bands are present at lower effective temperatures (and higher gravities). The band intensities alone are not, however, good measures of the temperatures since they are also highly sensitive to metallicity (and gravity). Better measures of the effective temperatures are obtained by the lunar occultation method (e.g., Richichi et al. 1992), interferometry (McAlister 1985, Quirrenbach et al. 1993), or the infrared flux method (Blackwell et al. 1991, Tsuji 1981a,b). Due to strong molecular absorption, the colours of cool stars are usually very far from a black body energy distribution, and effective temperatures can therefore only be extracted from colours by careful calibration to model-atmosphere predictions (e.g., Bell \& Gustafsson 1989, Buser \& Kurucz 1992, Fluks et al. 1994).

In practice a large problem with determination of the fundamental stellar parameters $\left(\mathrm{T}_{\text {eff }}, \log (g)\right.$, mass, and chemical abundance), is that all the spectral features are more or less sensitive to all of the parameters, and it is therefore difficult to find specific bands which can be used as index for grav- 
ity or temperature or chemical abundance alone. On the other hand, this "multi-response" makes it, at least in principle, possible to make a best fit to all parameters simultaneously, once high signal-to-noise, high-resolution spectra are obtained, and a grid of high-quality model atmospheres are computed. A nice step in that direction was recently taken by Cayrel et al. (1991), who computed a large grid of model atmospheres and, by use of a least square algorithm, determined best-fit model spectra to observed spectra. In this way they semi-automatically estimated $\mathrm{T}_{\text {eff }}, \log (\mathrm{g})$, and $[\mathrm{M} / \mathrm{H}]$ simultaneously for a sample of 41 stars. With the rapid development of neural networks and pattern recognition codes, such methods are promising for future semi-automatic determinations of fundamental parameters (and abundances) of very large samples of stars.

40 neutral diatomic molecules, 3 "molecular" ions (including $\mathrm{H}^{-}$), and 8 polyatomic molecules are known from photospheric spectra of cool stars including the solar photosphere (spectral type G2V) and sunspots (spectral type $\approx \mathrm{K} 5 \mathrm{~V}$ ). Table 1 lists them together with the type of stars in which they have been identified (or suggested). The table lists all the molecules which are "generally accepted" as identified, and it excludes all molecules which have been suggested in the literature but which are "generally believed" to be based on erroneous or very doubtful identifications. In between these two "concepts" is a grey land of likely, possible, slightly doubtful, more doubtful, etc. identifications, and in all these cases the table does not represent an objective grading of each single investigation, but merely my subjective impression of the quality of the identification based on discussions with numerous colleagues over the years. Molecules which are in this "gray" zone include $\mathrm{BO}, \mathrm{CrO}, \mathrm{CrH}, \mathrm{MgO}, \mathrm{SiF}, \mathrm{SiN}, \mathrm{ZnH}, \mathrm{CH}^{+}$, and $\mathrm{C}_{2} \mathrm{H}$ (which have been included), and $\mathrm{CoH}, \mathrm{FeO}, \mathrm{GeO}, \mathrm{HS}, \mathrm{MgF}$, and $\mathrm{H}_{2}^{-}$ (which have not been included in the table). Extensive references to the discovery papers and further observations of the listed molecules can be found in the reviews by Spinrad \& Wing (1969) and by Tsuji (1986), in the books by Jaschek \& Jaschek $(1987,1995)$, or in the paper by Davis (1947). Systematic updating of the references to the literature about these and other small molecules of astrophysical interest are given in the bi-monthly Berkeley Newsletter (Davis 1994).

\section{Available molecular data for stellar astrophysics}

Synthetic spectrum computation often requires high precision in the line frequencies (as well as in the line strengths) as opposed to the data used for the underlying model-atmosphere calculation, where completeness in the number of included lines is often more important than very high accuracy in the line frequencies and strengths. For synthetic-spectrum calculations, one 
TABLE 1. The 40 diatomic, 8 polyatomic, and 3 ionic molecules observed in photospheres of M, S, and C stars and the Sun (photosphere + sunspots).

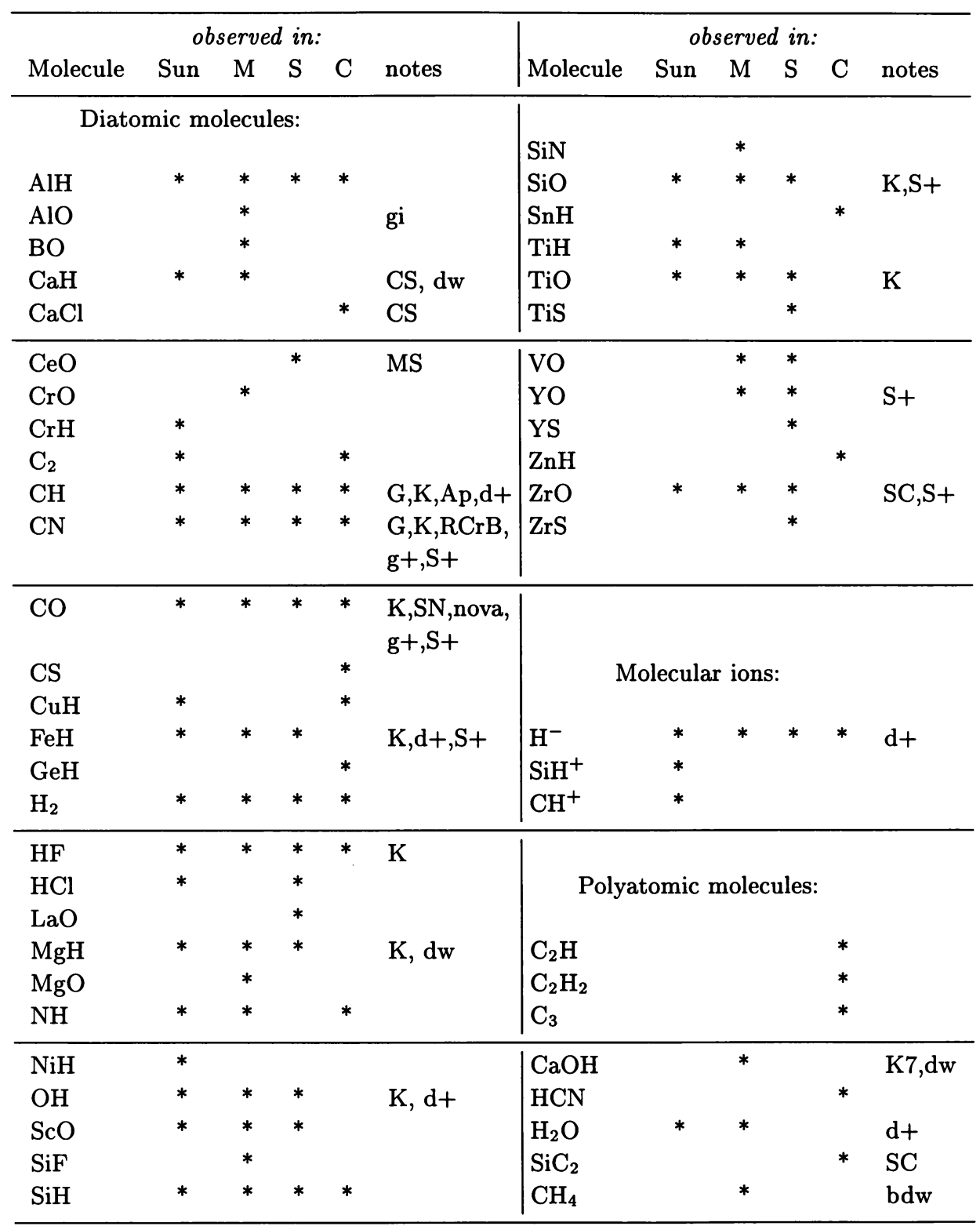

Notes: gi, dw means that the molecule has been observed only in giants or dwarfs, respectively. $g+$ and $d+$ refers to the bands of the molecule being stronger in giants than in dwarfs $(\mathrm{g}+)$ or visa versa $(\mathrm{d}+) . \mathrm{S}+$ refers to the bands being stronger in $\mathrm{S}$ stars than in $\mathrm{M}$ type giants. G, K, SC, CS, MS, Ap, RCrB, SN (super nova), and nova, respectively, refers to the molecule having been identified also in these types of stars. The note bdw for $\mathrm{CH}_{4}$ reminds us that $\mathrm{CH}_{4}$ has been observed not in "real M-type dwarfs" but in Brown Dwarfs only. 
therefore often needs to use directly the laboratory measurements rather than computed or interpolated data. The three most frequently used laboratory data bases are the HITRAN data base (Rothman et al. 1992; a high temperature version is in preparation), the GEISA data base (Husson et al. 1991), and the Berkeley Data Base (Davis 1994). The 1991/92 versions of the HITRAN and GEISA data bases contain frequency, line strength, excitation energies, and other data for about 700,000 lines from 39 molecules. The molecules are selected for their relevance for geophysical research, but the following 8 molecules from the two data bases have also been identified in stars (see Table 1): $\mathrm{CO}, \mathrm{HF}, \mathrm{HCl}, \mathrm{OH}, \mathrm{HCN}, \mathrm{C}_{2} \mathrm{H}_{2}, \mathrm{H}_{2} \mathrm{O}$, and $\mathrm{CH}_{4}$. The Berkeley Data Base was created specifically with the aim of providing high quality spectral line data for molecules of stellar interest, and it includes data on frequencies of lines from the following 6 molecules: $\mathrm{CN}, \mathrm{FeH}, \mathrm{LaO}$, $\mathrm{TiO}, \mathrm{YO}$, and $\mathrm{ZrO}$.

In total, the 3 laboratory data bases contain information for $\approx 100,000$ spectral lines from 14 molecules of interest for stellar astrophysics. These lists form a very important basis for identification and analysis of high resolution stellar spectra. However, stellar spectra contain a huge number of weak molecular lines as well, which are too numerous and/or too weak (at room temperature) to be measured in the laboratory. These lines are either from hot bands populated only at the relatively high temperatures in stellar atmospheres, or from highly excited rotational states. Typically, millions of such lines from each molecule contribute to the stellar opacity. Eriksson et al. (1984) and Jørgensen (1994b) demonstrated that the structure of the atmosphere of carbon-rich and oxygen-rich red giants are governed not by the strong lines, but mainly by a veil of $\approx 10$ million weak spectral lines from $\mathrm{HCN}$ and from $\mathrm{H}_{2} \mathrm{O}$, respectively.

The weak lines typically form a pseudo-continuous absorption in the stellar spectrum, and hence are not observable as individual lines, but their depression of the spectrum is often substantial. Plez et al. (1993) demonstrated that $70 \%$ of the flux in the region around the $\lambda 6707 \AA \mathrm{Li}$ line in the spectrum of red giants, is blocked by the pseudo-continuous veil of $\mathrm{TiO}$ molecular lines. The $\mathrm{Li}$ abundances derived respectively with and without the veil of $\mathrm{TiO}$ lines included in the surrounding spectral region are very different from one another, and the value is crucial for the understanding of stellar evolution as well as for cosmological models.

Thus, the 100,000 spectral lines from laboratory measurements represent only about $1 \%$ of the lines necessary for calculating a proper model atmosphere and for drawing a proper continuum in synthetic spectra. They serve mainly the (very important) basis for line identification in stellar spectra and for construction of complete ab initio line lists. Such computed lists exist today for approximately 100 million spectral lines. The agreement be- 
tween computed and measured line (/band) strengths for the strongest bands is generally excellent. For the weak bands the agreement is less satisfactory, but it is at present not obvious whether the computations or the measurements are most reliable.

The line frequencies listed in the "computed" data bases are generally not the computed ones, but the far better ones obtained from polynomial fits to measured frequencies. The methods used for the various lists (which vary considerably in quality and in degree of completeness) have been reviewed recently by Jørgensen (1995).

The SCAN data base contains information about identification, frequency, excitation energy, and strength for $\approx 70$ million lines from the molecules $\mathrm{CN}, \mathrm{CH}, \mathrm{TiO}, \mathrm{H}_{2} \mathrm{O}, \mathrm{HCN}, \mathrm{C}_{2} \mathrm{H}_{2}$, and $\mathrm{C}_{3}$. Most of the data is based on ab initio computations and represents a high degree of completeness in the number of included lines. The construction of the line lists and some application to stellar astrophysics have been described in detail in the literature (CN: Jørgensen \& Larsson 1990, CH: Jørgensen et al. 1996, TiO: Jørgensen 1994c, $\mathrm{H}_{2} \mathrm{O}$ : Jørgensen \& Jensen 1993, HCN: Jørgensen et al. 1985, $\mathrm{C}_{3}$ : Jørgensen et al. 1989). Most of the data are obtainable by anonymous ftp (on machine stella.nbi.dk, disk-area pub/scan; use userid anonymous and let password be your email address). Also the line lists of $\mathrm{CO}, \mathrm{C}_{2}$ and $\mathrm{CN}$ due to Querci et al. (1974) are now available via ftp at the same address, as is the absorption coefficient of pre-solar diamond grains extracted from carbonaceous chondrites (Andersen et al. 1996). When you access this disk area, you can find a description of how to use the individual line lists in the files "molecule.tex" (for example CH.tex) or "molecule.ps", and the data are generally in files "molecule.dat".

The Harvard-Smithsonian data base on atomic and molecular lines contains computed data for 15 million spectral lines from the 11 molecules $\mathrm{TiO}$, $\mathrm{C}_{2}, \mathrm{SiO}, \mathrm{CN}, \mathrm{CO}, \mathrm{H}_{2}, \mathrm{CH}, \mathrm{NH}, \mathrm{OH}, \mathrm{MgH}$, and $\mathrm{SiH}$. The original computations were described by Kurucz \& Peytremann (1975), and the present version was reviewed recently by Kurucz (1994). The lists are available on CD-ROMs from R. L. Kurucz.

Other frequently used line data for molecular opacity computations in stellar atmospheres include the list by Littleton \& Davis (1985) of 330,000 lines from $\mathrm{ZrO}$ and 1,200 lines from YO, the list by Tipping \& Chakerian $(1981)$ of $14,000 \mathrm{SiO}$ lines, the list by Goorvitch \& Chakerian (1994) of 35,000 CO lines, the list by Pineiro et al. (1987a) of 35,000 CS lines, the list by Pineiro et al. (1987b) of $620 \mathrm{SiS}$ lines. Two very recent lists by Langhoff \& Bauschlicher (SiO: 1994, TiO: 1996, in preparation), based on ab initio calculations and a high degree of completeness, are described elsewhere in this book, and a list of $\mathrm{H}_{2} \mathrm{O}$ lines under construction is described by Miller et al. (1994). 


\section{Cool giant stars}

Recent progress in modelling cool star atmospheres by use of molecular data has taken place for a wide range of stellar types. For the cool giants recent efforts include steps toward constructing unified model atmospheres covering the photosphere, the chromosphere, the wind, and the circumstellar envelope in a self-consistent treatment. One of the main problems in combining the existing fragments of such a unified self-consistent atmospheric model is the different treatment of the radiative transfer in the different parts of the atmosphere. In the photospheric models, the line list data described above are converted to opacity data by folding the individual lines with a profile and multiplying the results with the relevant partial pressures in each layer of the model, through each iteration of the model atmosphere computation. These individual opacities are then summed for all species, and the radiative transfer is solved for a statistically significant number of frequency points (typically $\approx 10,000$ frequencies). This technique, called the Opacity Sampling method, is today the most widely used for solution of the model photosphere problem, when many spectral lines, as the millions of molecular lines described above, are involved. The radiative transfer computation at the many frequencies is, however, so time consuming that it is necessary to impose other simplifications in order to solve the problem at a present-day computer. One of these limitations is the assumption of hydrostatic equilibrium, which obviously inhibits computations of dynamic (i.e., wind) properties.

On the other hand, the wind models demand so much computing time in solving the hydrodynamic problem, that the radiative transfer has to be treated very approximately. Usually, a constant absorption coefficient independent of frequency is assumed. An important step forward in solving this dilemma was recently taken by Helling (1996) who investigated how small a number of sampling points it is possible to chose (and where to chose them) in order to still get significantly better models than by use of various mean opacities (computed based on the same molecular line data). The results are shown in Fig. 1. The promising conclusion with regard to the possibility of constructing a unified model atmosphere, is that significant improvement over mean-opacity models can be achieved by use of relatively few opacity sampling points. It is also seen that, for the model illustrated here, the error by using a Rosseland mean, or a typical constant-kappa absorption coefficient, instead of full opacity sampling treatment, amounts to almost $1,000 \mathrm{~K}$ in the derived surface temperature of the model. Truly, such an error will predict a completely different chemistry and spectrum of a given model, or, alternatively, a completely different model fit to a given observed spectrum. 


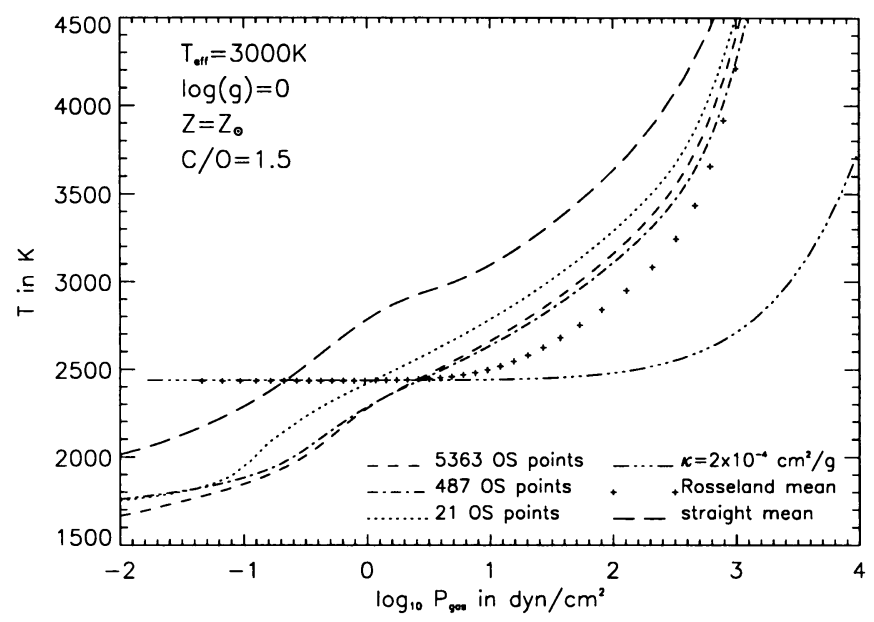

Figure 1. The temperature versus gas pressure structure of the atmosphere of a carbon star with $T_{\text {eff }}=3000 \mathrm{~K}, \log (g)=0.0, \mathrm{Z}=\mathrm{Z}_{\odot}$, and $\mathrm{C} / \mathrm{O}=1.5$. Except for the $\kappa=$ constant model, all models are computed based on the same opacity data, but sampled differently.

Hubble Space Telescope observations of chromospheric spectral lines from oxygen-rich giants as well as from carbon-rich ones, have provided new constraints on the temperature, gas pressure, and velocity conditions in the upper atmosphere (Johnson et al. 1995). Recent progress in the construction of chromospheric models of cool stars has been reviewed by Luttermoser (1996), and the status of our knowledge about the circumstellar envelope of cool stars is described by Olofsson elsewhere in this book.

Grain formation and wind models have successfully simulated flux distributions and light curves of very cool stars and extended atmospheric regions (e.g., Sedlmayr 1994, Dorfi \& Höfner 1996). Analysis of pre-solar grains from meteorites (e.g., Anders \& Zinner 1993, Dorschner \& Henning 1995) give us valuable and very detailed new knowledge about stellar dust grains, which we would not have been able to obtain from spectroscopic observations of stars (e.g., size distributions, grain forms, isotopic ratios, impurities, etc). However, it has not been possible to benefit efficiently from these new data in the modelling of stellar atmospheres. The reason is a lacking knowledge of the detailed formation processes (e.g., which routes of formation from molecules via clusters to grains, reaction time-scales, etc), a lack of understanding in which stellar types the most abundant pre-solar grains form (Jørgensen \& Andersen 1996), and a lack of unified cool-star atmospheric models.

In order to form grains, molecules must grow to macro-molecules, which 
eventually will cluster and become the nucleation seeds of the grains. However, this molecular part of the grain formation process is still only poorly understood, and the standard assumption (for the formation of carbonrich grains) is not straightforwardly applicable to stellar conditions. Since long, the seeds of the carbon-rich grains were speculated to originate via a clustering of Polycyclic Aromatic Hydrocarbon (PAH) molecules. Detailed modelling has, however, revealed that the PAH molecules themselves are unable to form under the conditions in standard stellar photospheric models (Helling et al. 1996) as well as in models of the wind (Frenklach \& Feigelson 1989). The (hydrostatic) photospheric models do not reach into the right temperature-pressure region of PAH formation in chemical equilibrium, and in the (dynamical) wind models the timescale for the gas in the favourable region is shorter than the timescale for $\mathrm{PAH}$ formation. A recent attempt to investigate if the nucleation problem can be solved by introducing appropriate shocks in the wind models, is presented by Cherchneff in this book.

\section{Cool dwarf stars}

A rapid expansion of the observational capacities in the infrared has been a major motivation for an intensified effort to model low luminosity cool stars - a tendency that recently experienced a new boost with the first observation of a Brown Dwarf (Nakajima et al. 1995). For the coolest dwarf stars the well known balance between carbon molecules and oxygen molecules breaks down. In cool giants all carbon atoms or all oxygen atoms (whichever are least abundant) are bound in carbon monoxide. Usually, the (sometimes small amount of) excess oxygen or carbon atoms relative to those bound in $\mathrm{CO}$ will form the molecules which are responsible for the bulk of the opacity. In the coolest dwarfs, however, $\mathrm{CH}_{4}$ molecules become more abundant than $\mathrm{CO}$, resulting in a more stable condition where a strong absorber $\left(\mathrm{CH}_{4}\right)$ is one of the most abundant molecules, too.

In a series of recent papers, Tsuji and collaborators analysed the changing structure of successively cooler dwarf models. Tsuji (1994) and Tsuji \& Onaka (1995) demonstrated the increasing importance of $\mathrm{H}_{2} \mathrm{O}$ and, in particular, of $\mathrm{CH}_{4}$ in the coolest dwarfs. These models predict, however, $\mathrm{H}_{2} \mathrm{O}$ bands too strong compared to observations, and considerably better agreement was reached by Tsuji et al. (1996a) by introducing also grain formation (of $\mathrm{Al}_{2} \mathrm{O}_{3}, \mathrm{Fe}$, and $\mathrm{MgSiO}_{3}$ ) in the models. The main simplifications in these models which still needs to be justified or improved upon, is the use of a smeared-out absorption coefficient (Tsuji 1994) for the molecular opacities and a simplified description of the grain formation (everything above the saturated vapor-pressure condense into single-sized grains).The 


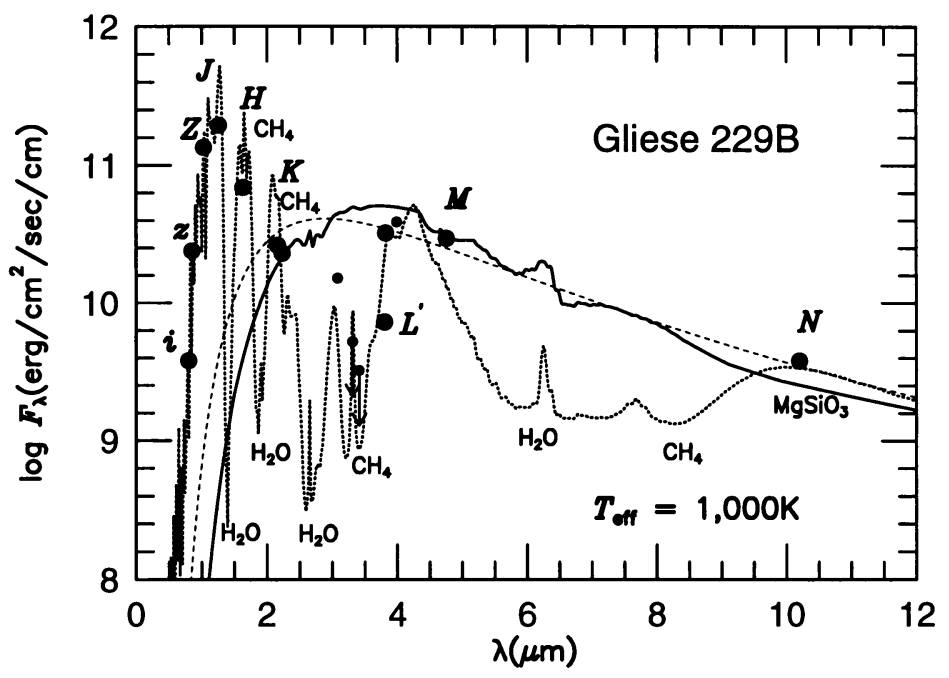

Figure 2. The observed spectral energy distribution (dots) of the cool Brown Dwarf GL 229B together with model spectra computed respectively with (full drawn line) and without (dotted line) the inclusion of dust. Bands of $\mathrm{H}_{2} \mathrm{O}$ and $\mathrm{CH}_{4}$ are indicated. Adapted from Tsuji et al. (1996b).

good agreement obtained between the observed and computed spectrum of the very cool dwarf LHS 2924 (spectral type M9V) is nevertheless impressive, and it is the first time detailed fully self-consistent $M$ dwarf models based directly on laboratory chemical input for molecules and grains have been constructed and compared successfully to observations.

While the importance of the water opacity for cool stars has been known for a long time both observationally (e.g., Jones et al. 1994) and theoretically (e.g., Jørgensen 1994b), the confirmation of the presence of $\mathrm{CH}_{4}$ by the spectroscopic identification in the Brown Dwarf Gl229B (Oppenheimer et al. 1995) is new. Dust-free models of this object were constructed by Allard et al. (1996) and by Tsuji et al. (1996b). While Tsuji et al. (1996b) showed that dust-free models fit the observed spectrum of this Brown Dwarf better than corresponding models with dust included in the equilibrium (see Fig. 2), this fact leaves open the question of why dust seems to be present in the spectrum of cool M dwarfs, but not in the even cooler object Gl 229B where the conditions for dust formation should be expected to be even more favourable.

M dwarfs (and Brown Dwarfs) have a lifetime considerably longer than the present age of the Universe, and the oldest such objects can therefore give us important information about the formation of our Galaxy (and the Universe) if we are able to model them well (and to observe them). 


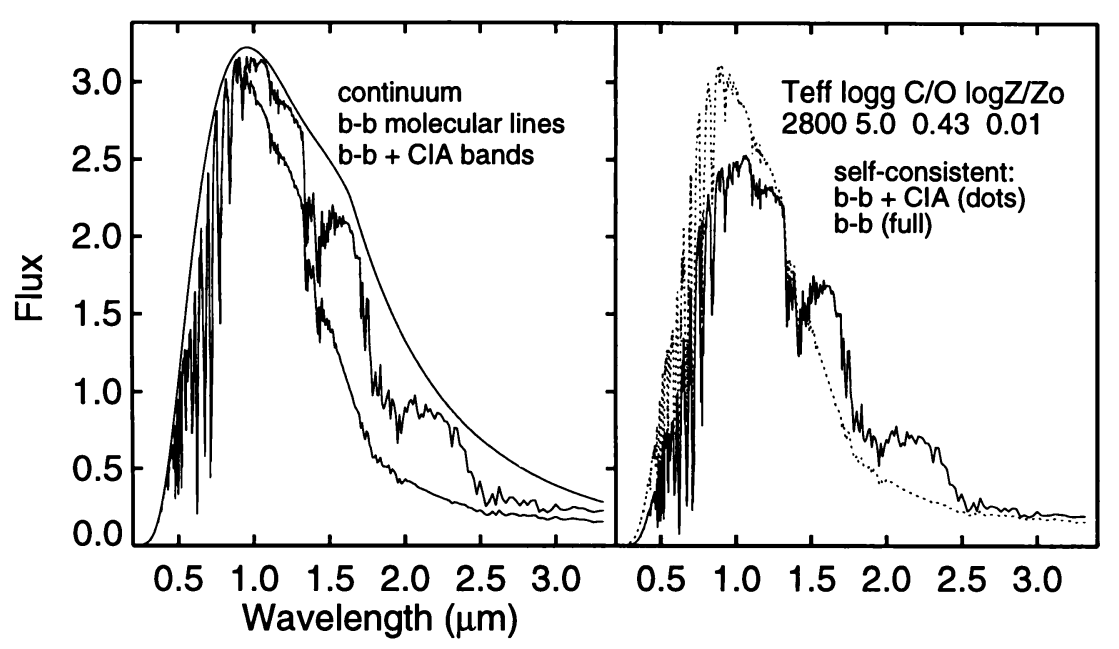

Figure 3. The contributions of various opacity sources to the spectrum are shown in the left panel for a stellar model with $\mathrm{T}_{\text {eff }}=2800 \mathrm{~K}, \log (\mathrm{g})=5.0$, and $\mathrm{Z}=10^{-2} \mathrm{Z}_{\odot}$ - a typical main-sequence member of a globular cluster. The model computations include opacities of continuum sources, molecular lines, and CIA. The spectra are computed based on respectively the continuum alone (upper, convolving curve), continuum + molecular $(b-b)$ lines, and continuum + molecular lines + CIA. Only the latter is consistent with the underlying model atmosphere, but the difference between the three spectra illustrates the relative contribution of the three sources of opacity. In the panel to the right self-consistent spectra based on corresponding models with and without CIA, respectively, are shown.

While dust and molecular $b-b$ transitions dominate the opacities in the spectrum of the solar metallicity M dwarfs and Brown Dwarfs, the question is to what degree these opacity sources will still be important when we become able to observe the dwarfs that formed in the very beginning of the Galactic history. Such stars will predominantly be found in the globular clusters and in the Galactic halo, and are spectroscopically yet a bit below our observational capabilities. With decreasing metallicity (i.e., primarily decreasing abundances of $\mathrm{C}, \mathrm{N}$, and $\mathrm{O}$ ) the abundance of the "normal" polar molecules decreases correspondingly, and collision induced absorption (CIA) in pairs of non-polar molecules can be expected to dominate over dipole absorption of single polar molecules.

Borysow (1994) reviewed the physics of collision induced absorption processes, and Borysow et al. (1996) investigated for which region of the HR diagram (stellar effective temperature, gravity, and metallicity) CIA dominates the structure and spectrum. An example from this investigation is shown in Fig 3, for a model representing a typical Mdwarf star in a globular cluster. It is seen that the model spectrum is quite featureless in 
the entire infrared region when CIA is included, because of the dominant and pseudo-continuous character of this absorption mechanism. The effect is largest for cool, low metallicity dwarf stars, but it affects the spectrum even for cool metal-poor stars with gravity as low as $\log (\mathrm{g})=2$ (i.e., subgiants), for metal-poor dwarfs as warm as $4000 \mathrm{~K}$, and for cool dwarfs with metallicity as high as $\mathrm{Z}=0.1 \mathrm{Z}_{\odot}$. We predict that the estimated effective temperature will be lower, and the metallicity higher, when comparisons of observed spectra are done with spectra based on model atmospheres where a proper treatment of CIA is counted for than if interpreted without this opacity source, as is illustrated in the right panel of Fig 3.

\section{Acknowledgements}

I am thankful to C. Helling and to A. Borysow for letting me include respectively Fig 1 and Fig 3 prior to publication, and to T. Tsuji for providing me with a postscript version of the original of Fig. 2. Valuable comments from H.R. Johnson are gratefully acknowledged. This work was supported by the Danish Natural Science Research Council.

\section{References}

Allard F., Hauschild P.H., Baraffe I., Chabrier G. 1996, ApJ 465, L123

Anders E., Zinner E. 1993, Meteoritics 28, 490

Andersen A.C., Jørgensen U.G., Nicolaisen F., Sørensen P.G., Glejbøl K. 1996, A\&A, in press

Bell R.A., Gustafsson, B., 1989, MNRAS 236, 653

Bessell M.S., Brett J.M. 1988, PASP 100, 1134

Bonnell J.T., Bell R.A. 1993, MNRAS 264, 319; MNRAS 264, 334

Blackwell D.E., Petford A.D., Shallis M.J., 1980, A\&A, 82, 249

Borysow A. 1994, in: U.G.Jørgensen (ed.) 1994a, p. 209

Borysow A., Jørgensen U.G., Zheng C. 1996, A\&A, submitted

Buser R., Kurucz R.L. 1992, A\&A 264, 557

Cayrel R., Perrin M.-N., Buser R., Barbuy B. \& Coupry M.-F. 1991, A\&A 247, 122

Davis D.N. 1947, ApJ 106, 28

Davis, S.P., 1994, in: U.G.Jørgensen (ed.) 1994a, p. 397

Dorfi E., Höfner S. 1996, in: R.F.Wing (ed.) 1996

Dorschner J., Henning T. 1995, A\&A Rev. 6, 271

Eriksson K., Gustafsson B., Jørgensen U.G., Nordlund A. 1984, A\&A 132, 37

Frenklach M., Feigelson E.D. 1989, ApJ 341, 372

Fluks M.A., Plez B., The P.S., deWinter D., Westerlund B.E., Steenman H.C. 1994, A\&AS 105,311

Goorvitch D., Chackerian C. Jr. 1994, ApJS 91, 483

Gustafsson B., 1989, ARAA 27, 701

Gustafsson B., Jørgensen U.G., 1985, In: Calibration of Fundamental Stellar Quantities, D.S.Hayes et al. (eds.), p. 303

Gustafsson B., Jørgensen U.G. 1994, A\&A Rev., 6, 19

Helling C. 1996, Diploma Thesis, Technical University Berlin

Helling C., Jørgensen U.G., Plez B., Johnson H.R. 1996, A\&A 315, 194 
Husson N., Bonnet B., Scott N.A., Chedin A., 1991, Internat Note, Laboratoire de Metéorologie Dynamique. No. 163

Jaschek C., Jaschek M. 1987, The Classification of Stars, Cambridge Univ. Press

Jaschek C., Jaschek M. 1995, The behavior of chemical elements in stars, Cambridge University Press, Cambridge

Johnson H.R., 1986, In: The M-type Stars, H.R.Johnson and F.R.Querci (eds.), NASA SP-492, p. 323

Johnson H.R. 1991, In: Stellar Atmospheres: Beyond Classical Models, Crivellari (ed.)

Johnson H.R. et al. 1995, ApJ 443, 281

Jones H.R.A., Longmore A.J., Jameson R.F., Mountain C.M. 1994, MNRAS 267, 413

Jørgensen U.G. 1995, In: ASP Conf. Ser. vol. 78, S.J.Adelman, W.L.Wiese (eds.), p. 179

Jørgensen U.G. (ed.) 1994a, Molecules in the Stellar Environment, Lecture Notes in Physics vol. 428, Springer-Verlag

Jørgensen U.G. 1994b, In: Jørgensen U.G. 1994a, p. 29

Jørgensen U.G. 1994c, A\&A, 284, 179

Jørgensen U.G. 1992, Rev. Mexicana Astron. Astrof. 23, 195

Jørgensen U.G., Almlöf J., Gustafsson B., Larsson M., Siegbahn P. 1985, J. Chem. Phys. 83,3034

Jørgensen U.G., Almlöf J., Siegbahn P.E.M. 1989, ApJ 343, 554

Jørgensen U.G., Andersen A. 1996, in: R.F.Wing (ed.) 1996

Jørgensen U.G., Jensen P. 1993, J. Mol. Spectrosc. 161, 219

Jørgensen U.G., Larsson M. 1990, A\&A 238, 424

Jørgensen U.G., Larsson M., Iwamae A., Yu B. 1996, A\&A 315, 204

Jørgensen U.G., Wing R.F. 1996, in: R.F.Wing (ed.) 1996

Kurucz R.L., Peytremann E. 1975, SAO Spec. Rept., 362

Kurucz R.L., 1994, in: U.G.Jørgensen (ed.) 1994a, p. 282

Langhoff S.R., Bauschlicher C.W. 1994, Chem. Phys. Lett. 211, 305

Littleton J.E., Davis S.P. 1985, ApJ, 296, 152

Luttermoser D.G. 1996, in: R.F.Wing (ed.) 1996

Martin W.C., 1992, In: Atomic and Molecular Data for Space Astronomy, Lecture Notes in Physics vol. 407, P.L. Smith\& W.L. Wiese (eds.) (Berlin:Springer), p. 121

McAlister H.A. 1985, ARAA 23, 59

Miller T., Tennyson J., Jones H.R.A., Longmore A.J. 1994, in: U.G.Jørgensen (ed.) 1994a, p. 296

Nakajima T., Oppenheimer B.R., Kulkarni S.R., Golimowski D.A., Matthews K., Durrance S.T. 1995 , Nature 378,463

Oppenheimer B.R., Kulkarni S.R., Matthews K., Nakajima T. 1995, Science 270, 1478

Piñeiro, A.L., Tipping, R.H., Chackerian, C.Jr. 1987a, J. Mol. Spec. 125, 91

Piñeiro, A.L., Tipping, R.H., Chackerian, C.Jr. 1987b, J. Mol. Spec. 125, 184

Plez B., Smith V.V., Lambert D.L., 1993, ApJ, 418, 812

Querci F., Querci M., Tsuji T., 1974, A\&A, 31, 265

Quirrenbach A., Mozurkewich D., Armstrong J.T., Buscher D.F., Hummel C.A. 1993, ApJ 406, 215

Richichi A., DiGiacomo A., Lisi F., Calamai G. 1992, A\&A 265, 535

Rothman L.S., et al. 1992, JQSRT, 48, 469

Sauval A.J., Blomme R., Grevesse N. (eds.) 1995, Laboratory and Astronomical High Resolution Spectra, ASP Conference Series vol. 81

Sedlmayr E. 1994, in: U.G.Jørgensen (ed.) 1994a, p. 163

Spinrad H., Wing R.F. 1969, ARA\&A 7, 249

Tinney C.G. (ed.) 1995, The Bottom of the Main Sequence and beyond, ESO Conf. Ser.

Tipping R.H., Chackerian C.Jr. 1981, J. Mol. Spec. 88, 352

Tsuji T., 1981a, A\&A 99, 48

Tsuji T., 1981b, J. Astrophys. Astron. 2, 95

Tsuji T. 1986, ARAA, 24, 89

Tsuji T. 1994, in: U.G.Jørgensen (ed.) 1994a, p. 79 
Tsuji T., Ohnaka K. 1995, In: Elementary Processes in Dense Plasmas, S.Ichimaru, S.Ogata (eds.), Addison-Wesley

Tsuji T., Ohnaka K., Aoki W. 1996a, A\&A 305, L1

Tsuji T., Ohnaka K., Aoki W. 1996b, A\&A 308, L29

Wing R.F. (ed.) 1996, IAU Symp. 177, The Carbon Star Phenomenon, Kluwer, Dordrecht 